\title{
Look up: Human adults use vertical height cues in reorientation
}

\author{
Yu Du ${ }^{1} \cdot$ Marcia L. Spetch $^{1} \cdot$ Weimin Mou ${ }^{1}$
}

Published online: 17 June 2016

(C) Psychonomic Society, Inc. 2016

\begin{abstract}
Numerous studies have shown that people and other animals readily use horizontal geometry (distance and directional information) to reorient, and these cues sometimes dominate over other cues when reorienting in navigable environments. Our study investigated whether horizontal cues (distance/angle) dominate over vertical cues (wall height) when they are in conflict. Adult participants learned two locations (opposite corners) in either a rectangular room (with distance information) or a rhombus room (with angle information). Both training rooms had 2 opposite high walls as height cues. On each trial, participants were disoriented and then asked to locate the correct corners. In testing, the rooms were modified to provide (a) distance or angle cues only, (b) height cues only, and (c) both height and horizontal cues in conflict. Participants located the correct corners successfully with horizontal (distance/angle) or height cues alone. On conflict tests, participants did not show preference for the horizontal information (distance/angle) over the height cues. The results are discussed in terms of the geometric module theory and the adaptive combination theory.
\end{abstract}

Keywords Spatial reorientation - Geometric cues $\cdot$ Height . Geometric module $\cdot$ Adaptive combination theory

Imagine that you travel in an underground subway train to an unfamiliar place. When you get off the train, step out of the subway station, and return to the world on the ground, the first

Marcia L. Spetch

mspetch@ualberta.ca

1 Department of Psychology, University of Alberta, P-217, Biological Science Building, Edmonton, Alberta, Canada T6G 2E9 thing you probably do is to figure out which direction is which, or to orient yourself. The ability to orient within an environment is essential for all mobile organisms, including humans. After a period of disorientation (e.g., traveling in an underground subway train), organisms need to reestablish their relations to the environment to navigate in a correct direction, which is called reorientation. During this process, many cues in the environment could potentially be used to reestablish bearings. For example, for hikers in natural environments, a mountain's peak is usually a salient cue for orientation because it can be seen from $100 \mathrm{~km}$ away, and it provides enduring and reliable information about directions in the environment. Mountains can also be seen as extended surfaces that form borders of a navigable environment. In this case, the geometric shape of the boundary of the environment formed by mountains could be used as an orienting cue. Indeed, the panoramic contour formed by the view of terrestrial objects against the skyline has been shown to be a potent navigational cue for foraging ants (e.g., Graham \& Cheng, 2009; Legge, Wystrach, Spetch, \& Cheng, 2014; Wystrach, Beugnon, \& Cheng, 2011).

Numerous studies have shown that the horizontal geometry of a navigable environment with continuous surfaces is a salient cue for reorientation in a variety of species (e.g., Cheng, 1986; for review, see Cheng, Huttenlocher, \& Newcombe, 2013; Cheng \& Newcombe, 2005). The pioneering study was conducted by Cheng (1986), who found that rats reoriented according to the geometric shape of an enclosed environment, sometimes ignoring features in the environment such as distinct visual patterns or odours. Cheng also introduced a paradigm that has become widely used for the study of reorientation, not only in rats but also in many other species including humans. Briefly, the subject first learns the location of a reward, which is usually at one corner of a room with geometric properties. The reward is then hidden and the 
subject is rotated to become disoriented before searching for the reward. Accurate search indicates successful re-orientation. Cheng found that rats searched not only at the correct location, but also at an incorrect but geometrically equivalent location (for review, see Cheng, Huttenlocher, \& Newcombe, 2013; Cheng \& Newcombe, 2005), indicating use of the geometric shape of the enclosure for reorientation.

In the first study with humans, Hermer and Spelke (1994, 1996) similarly found that children ages 1.5 to 2 years could use the geometric information of a rectangular room to locate the correct corner or its diagonally opposite corner that shared the same geometric information as the correct one (e.g., both corners had a longer wall on the left and a shorter wall on the right). These two studies also suggested that featural cues, such as a wall with a distinct color in a small room (6 ft by $4 \mathrm{ft}$ ), could not be used by children to distinguish the correct corner and its geometric equivalent until 6 years of age. To explain these surprising results, the geometric module account was proposed, suggesting an innate cognitive module that is specialized in processing geometric information in reorientation (Cheng, 1986; Hermer \& Spelke, 1994, 1996). In natural settings, the geometric properties of the environment made by mountains or shorelines are more stable and enduring in the long term compared to nongeometric surface properties (referred to as "featural cues"), such as leaf color, snow cover, or the presence of small stones, and therefore using the geometric properties is assumed to be adaptive.

Some subsequent studies, however, have provided evidence that opposes the geometric module theory (see Twyman \& Newcombe, 2010, for a review). For example, young children can use both geometric and nongeometric information to reorient in a larger space (Learmonth, Nadel, \& Newcombe, 2002; Learmonth, Newcombe, \& Huttenlocher, 2001; Learmonth, Newcombe, Sheridan, \& Jones, 2008) and in certain situations, the use of featural cues was observed in 1.5- to 2-year-old toddlers (Nardini, Atkinson, \& Burgess, 2008). Additionally, geometric information such as corner angles in a rhombus enclosure could not be used by children until 4 years of age (Hupbach \& Nadel, 2005), which is later than the age at which children can use the geometric shape of a rectangular room to orient.

To explain these findings, Newcombe and Huttenlocher (2006) proposed an alternative account, adaptive combination theory, which states that both geometric and nongeometric information can be used for reorientation depending on the circumstances. If the information is more salient, more reliable, more familiar, or more stable, then it is more likely to be taken into account than other competing sources of information (Twyman, Nardi, \& Newcombe, 2013). Therefore, geometric cues should not always be dominant in reorientation; instead, humans (and other species) should be flexible in using cues in reorientation.

In response to the evidence against the geometric module account, Spelke, Lee, and Izard (2010) proposed a modified geometric module theory. Their theory suggests a core geometric system for navigation in large-scale environments (here, the term large-scale environments is referring to navigable spaces, including small rooms); this core system operates from a very young age and captures only the distance (e.g., wall lengths between corners) and directional information (i.e., left/right sense) on extended surfaces (Spelke et al., 2010). Hence, according to Spelke et al. (2010), other geometric properties, such as angles, are not included in this core system, despite angle being a central Euclidean property. This assumption allows them to explain why the ability to use angle information was shown at a later age than distance and left/right sense (Hupbach \& Nadel, 2005). Furthermore, this theory attributes the failure of young children to use certain horizontal distance cues to the lack of threedimensional borders. For example, 3.5 -year-old children were able to use a rectangular frame that was $2-\mathrm{cm}$ high from the ground to reorient (Lee \& Spelke, 2011), but they could not use lines drawn on the floor, forming a same rectangular configuration (Lee \& Spelke, 2008). Similar results were also found in chicks (Lee, Spelke, \& Vallortigara, 2012).

Most previous studies on reorientation have focused only on the horizontal dimension of the environment. However, information from the vertical dimension should not be neglected. Terrain features such as a mountain's height or the slope of the ground can provide stable, reliable information that could guide three-dimensional navigation of mobile organisms. There is evidence that the vertical properties of terrain features can be used to reorient. For example, when tested in a square room with a tilted floor, where slope was the only available cue, human adults could use the tilted floor to reorient (Nardi, Newcombe, \& Shipley, 2011). Human children showed above-chance ability to use slope cue for reorientation at ages 8 to 10 years (Holmes, Nardi, Newcombe, \& Weisberg, 2015). Similarly, pigeons that were trained on a slope could encode a goal location based on the vertical and orthogonal (i.e., left/right) axes of the slope (Nardi \& Bingman, 2009; Nardi, Nitsch, \& Bingman, 2010).

Slope cues are complex because they can provide orienting information through visual, proprioceptive, and kinesthetic modalities and from both the horizontal and vertical dimensions (Hu, Zhang, Wu, \& Shao, 2015; Nardi et al., 2011). Only a few studies have explored the use of purely vertical information in humans. When 3.5-year-old children were presented with four identical boxes (for hiding target) forming a rectangular configuration on a circular carpet with two 10-cmhigh parallel bumps (which formed two borders of the rectangle), they could use such bumps to reorient (Lee \& Spelke, 2011). In contrast, children at this age could not use four 
identical objects, the 2-D form on the floor, or lines drawn on the floor to reorient, although these cues also provided the same rectangular configuration and horizontal distance cues as the bumps (Lee \& Spelke, 2008, 2011). However, Hu et al. (2015) found that children used the distance information from horizontal geometry at a younger age than the vertical distance information from wall height. In their study, 3- and 4-year-old children were tested in three different enclosures: a rectangular room with walls at the same height and a flat ceiling (where only geometric shape information was available), a square room with walls at different heights and a slanted ceiling (where only height cues were available), and a rectangular room with walls at different height and a slanted ceiling (where both geometric shape and height cues were available). $\mathrm{Hu}$ et al. (2015) found that both age groups could use geometric shape to find the hidden toy in the flat-ceiling rectangular room, but only 4-year-old children could use height to reorient in the two slanted-ceiling rooms. They suggested that the geometric shape of the environment takes priority over height cues during reorientation by children and that height may not be a component in the geometric module.

Numerous studies make clear that humans and other animals are capable of using several types of cues to orient within the environment. Given, however, that most natural environments contain multiple cues that could potentially be used for reorientation, an important question for both practical and theoretical reasons is how people weight different types of cues and why. Researchers have investigated the relative dominance of cues within the reorientation paradigm by training with more than one type of cue during the learning phase. In studies with human children, each cue type is then tested with the other cue(s) removed to determine whether it was learned and could be used to reorient. In this paradigm, differences in the age at which different cue types are learned have been taken as an indication of the cue's relative dominance. Another way to test the relative dominance is to conduct socalled conflict tests after the learning phase; on these tests, the environment is manipulated to place the cues in opposition to one another (e.g., after learning in a rectangular room with a red wall - a featural cue - on the short side of the room, in testing, the wall color is rotated to the long side of the wall, to place the featural cues of wall colors in opposition to the geometric cues of the room shape). The extent to which searching follows each cue type is taken as an indication of that cue's dominance.

Although the ability to use vertical cues for reorientation has now been established in humans and other animals, the relative priority of horizontal geometry and vertical cues has not been thoroughly studied. A few experiments in fish with different navigation tasks suggest that when cues were in conflict, the fish give more weighting on the vertical information than on the horizontal information, which is probably due to hydrostatic pressure (Holbrook \& Burt de Perera, 2009, 2011).
Some animal experiments suggest that slope, a type of cue containing vertical information, has priority over horizontal geometry. For example, when pigeons were trained in a sloped trapezoid arena and later tested with the geometric shape of the environment and the slope cue presented in conflict, they primarily used slope (Nardi \& Bingman, 2009), suggesting that pigeons rely on slope more heavily than horizontal geometry. A subsequent study showed that pigeons relied upon slope even when it was less predictive than horizontal geometry, and the authors argued that slope is given more weight because it is more salient (Nardi et al., 2010). The study by $\mathrm{Hu}$ et al. (2015), however, suggested that horizontal geometry (specified as distance and left/right sense) was dominant over height cues for reorientation by human children because it was learned at a younger age.

Our study was designed to examine the relative priority given by adult humans to vertical height cues when they are learned in conjunction with one of two kinds of horizontal cues: geometric distance cues provided by the shape of a rectangular room, or angular information from the corners of a rhombus room. In both cases, the use of height cues and the relative priority given to height cues was assessed by tests with cues presented in isolation and with cues presented in conflict. As previously discussed, there is ample evidence that geometric distance cues are a prominent cue for reorientation in many species, and these cues are assumed by Spelke and colleagues (2010) to be part of the core geometry system, whereas the role of angles in reorientation is less clear. Although adult humans can readily reorient by angular information (Lubyk, Dupuis, Gutiérrez, \& Spetch, 2012; Lubyk, Spetch, Zhou, Pisklak, \& Mou, 2013), Spelke et al. (2010) suggested that angular information is not part of the core system based on developmental data, and Sturz, Forloines, and Bodily (2012) have provided evidence suggesting that corner angles may function as featural cues. Thus, the priority given to height cues may differ depending on the type of horizontal cue that is concurrently available during the learning phase.

The height cues used in our study differed in two ways from those used in $\mathrm{Hu}$ et al. (2015). First, their study was conducted in rooms that had slanted ceilings, which meant that either the wall height or the angles formed by the ceiling and walls could potentially provide orienting cues, whereas our study was conducted in virtual rooms that had no ceilings and the height cues were provided purely by the height of the walls. Second, their height cues were asymmetrical with respect to horizontal geometry, such that the corners that were correct according to horizontal geometry were not identical in terms of height information. In our study, the opposite walls were at the same height and hence the height information and the horizontal cues were equally predictive of the correct locations (see Fig. 1). 

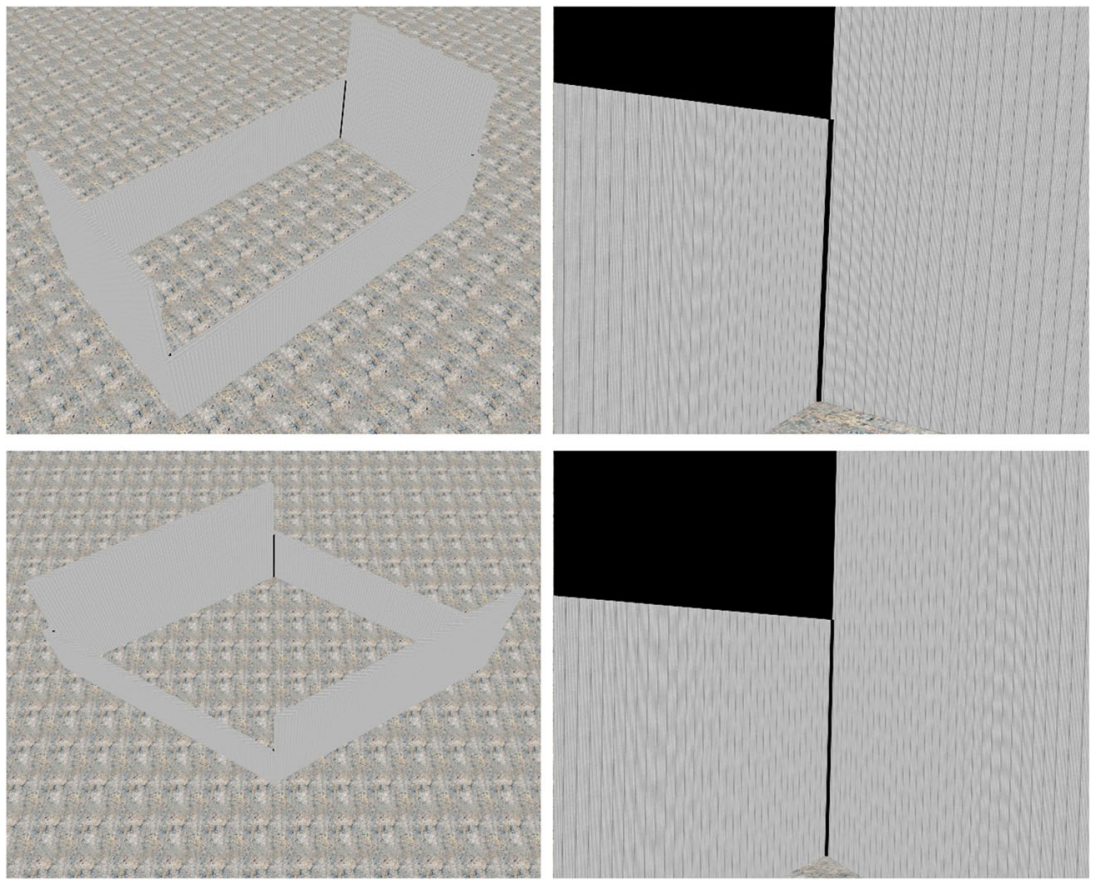

Fig. 1 Elevated plan of the rectangular (top) and rhombus (bottom) environments used for the training trials and Control test, and two examples of internal views (screen shot) from each environment. The viewpoint for the internal screen shots were from a person of $1.64-\mathrm{m}$

After the learning phase, participants were tested in modified environments that provided horizontal cues only, height cues only, or both cues presented in conflict (see Fig. 2). Because older children in Hu et al.'s (2015) study were able to use height information, and because we speculated the vertical height of extended surfaces should be a useful cue for reorientation in nature, we anticipated that adults would reorient well by height cues alone. However, because the younger children in Hu et al. (2015) used horizontal geometry but not height cues in the rectangular environment, we anticipated that horizontal distance information would dominate over the height information in conflict tests following learning in the rectangular environment. The relative priority of height cues over corner angles has never previously been tested, and therefore we had no basis for predicting which cue would dominate in the rhombus environment.

\section{Experiment 1}

\section{Method}

Participants Thirty-nine students (20 males, 19 females, age range $=17-24$ years, $M=18.97$ years) from the University of Alberta participated in Experiment 1. Eight additional participants were excluded because their performance on the Control test (see Fig. 2 and the following sections for details) was lower than $80 \%$ correct in the rectangular environment

height, wearing the helmet and standing in the center of the virtual room. Internal views vary as a result of horizontal and vertical movements of the participant's head

(six participants), the rhombus environment (one participant), or in both environments (one participant). This poor performance suggested that they had not adequately learned the correct corners and therefore made their test results difficult to interpret. All of the participants received course credits for their participation.

Apparatus The experiment was conducted in a 4-m $\times 4-\mathrm{m}$ room. Participants saw the experimental environments through a screen on a headset. The environments were presented by a virtual reality system that used Vizard software (WorldViz, Santa Barbara, CA), an nVisor head-mounted display (HMD, NVIS, Inc., Reston, VA), and an IS-900 motiontracking system. Screen resolution within the display was 1 , $280 \times 1,024$ pixels. The horizontal field of view (FOV) was $44^{\circ}$ and the vertical FOV was $35^{\circ}$. Participants' visual orientation was tracked by an InterSense (Billerica, MA) IS-900 motion-tracking system. To make a response, participants used an InterSense IS-900 Wand (InterSense, Inc., Billerica, MA), which controlled a long blue wand appearing in the virtual environment. Participants could move the wand to indicate their pointing and click a button on the wand to make a response.

Experimental design Participants were tested individually. Each participant received two sessions, each consisting of training trials followed by testing trials, but with differently shaped virtual training rooms (see Fig. 1). One session was 


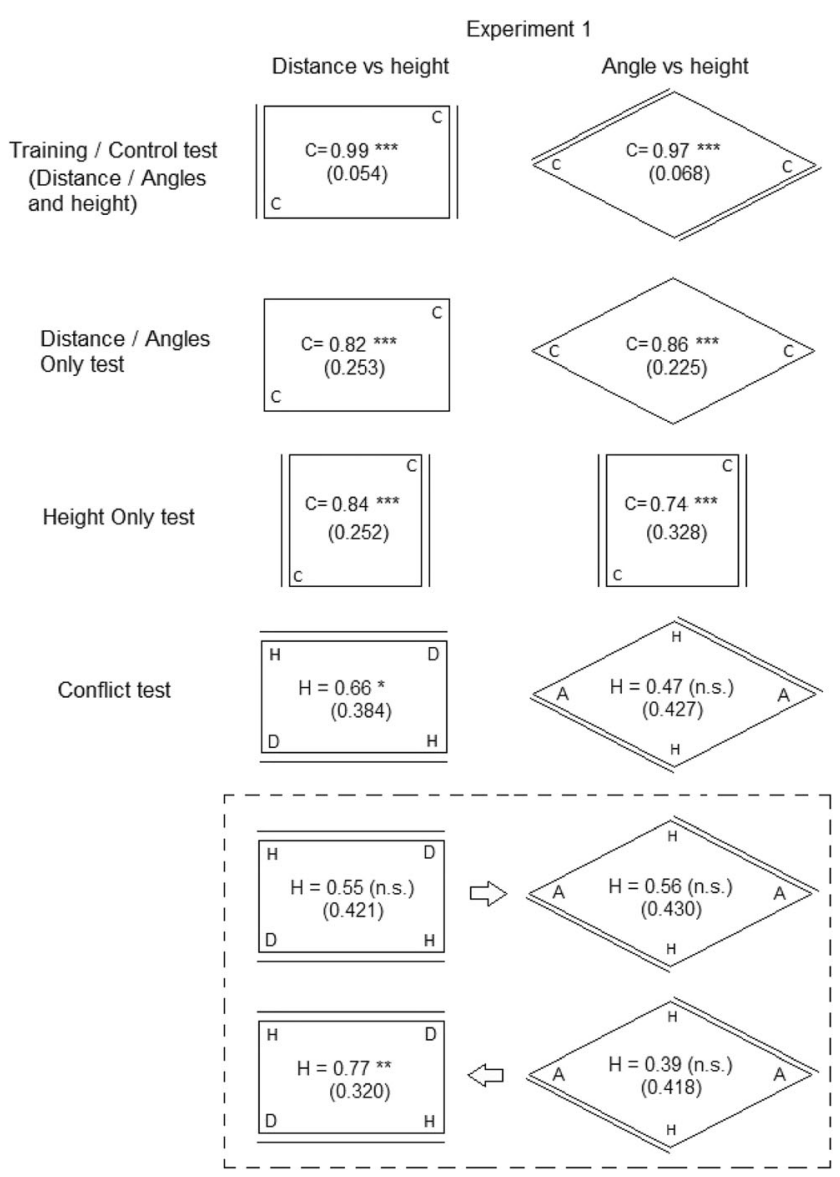

Fig. 2 The top view of the experimental environments and the average choice proportion in each test in Experiment 1 compared with chance level $(0.5)$ in each test (one-sample $t$ test: $* * * p<.001$ $<$ corrected $\alpha$; ${ }^{*} p<.05<$ corrected $\alpha$; n.s. $p>.05$ ). The double line indicates a high wall, and the single line indicates a low wall. The letter $C$ indicates a correct corner. In the Conflict test, the letter $H$ indicates a correct corner according to height cues. The figure inside the dashed lines illustrates the results in the Conflict test by session order. For simplicity, the correct corners for the rectangular environment are illustrated for the case in which the corners on the left of a short wall were correct, and for the rhombus environment, the acute corners were correct. The numbers in parentheses are standard deviation in that test

conducted in a rectangular room (rectangular environment), and the other session was conducted in a rhombus room (rhombus environment). The order was counterbalanced across participants. During the interval between the two sessions, which lasted for approximately 1 minute, participants were asked to close their eyes to avoid seeing stimuli other than the environments.

Experimental environments The experimental environments are shown in Fig. 1. In both training rooms, two opposite walls were twice as high $(3.6 \mathrm{~m})$ as the other two walls $(1.8 \mathrm{~m})$. For the rectangular environment, the training room was a $7.93-\mathrm{m} \times 3.97-\mathrm{m}$ rectangle, and the two $3.97-\mathrm{m}$ walls were the high walls; thus, the training room provided both distance information and height cues. For the rhombus environment, the training room was a $6.3-\mathrm{m} \times 6.3-\mathrm{m}$ rhombus, and the two walls to the right of the acute corners $\left(60^{\circ}\right)$ were the high walls; thus, these training rooms provided angle information and height cues. The two training rooms were the same overall size.

In the testing phase, for the rectangular environment, the tests were (1) a rectangular room identical to the training room (Control); (2) a rectangular room that maintained the same shape as the training room but with walls of equal height (i.e., with distance information only; Distance Only); (3) a square room with two opposite high walls that maintained the same size as the training room (i.e., with height cues only; Height Only); and (4) a rectangular room that maintained the same shape as the training room but with the high walls moved to the two long walls (i.e., high walls conflicted with the distance information; Conflict). For the rhombus environment, the testing environments were (1) a rhombus room identical to the training room (Control); (2) a rhombus room that maintained the same shape as the training room but with walls of equal height (i.e., with angular information only; Angles Only); (3) a square room with two opposite high walls that maintained the same size as the training room (i.e., with height cues only; Height Only); and (4) a rhombus room that maintained the same shape as the training room but with the high walls moved to be left of the acute corners (i.e., high walls conflicted with the angular information; Conflict).

Procedure Before the experiment, participants were informed that the experiment included two sessions, and in each session they were asked to first learn two correct corners of a room and later choose one correct corner. The participants were also told that the room would disappear and get rotated during intertrial intervals, and sometimes they would not receive feedback on their choice.

Each participant was led by an experimenter into the physical room with eyes blindfolded. After putting on the headset, participants saw the training room in a virtual environment, and they were standing at the center of the room. Participants were asked to look around freely and pay attention to the characteristics of the walls while maintaining a position at the center of the room.

Each session included one initial training trial, two training phases, and a testing phase. During each intertrial interval, the environment turned completely dark for approximately $5 \mathrm{sec}$ onds, then the room appeared again but in a randomly determined orientation. Participants were not necessarily facing the same orientation after the intertrial interval. Therefore, on each trial, participants needed to reorient themselves in the room.

On the initial training trial, participants used the wand to point to each of the four corners and click as a response. Following the clicking at each corner, a feedback sign with sound showed up to indicate whether the corner was correct. If 
the corner was correct, the feedback was positive - a gold coin appeared accompanied by a "ding" sound. If the corner was incorrect, the feedback was negative and showed an X accompanied by a jarring noise. The correct corners were always two diagonal corners. Across participants, the locations of correct corners were counterbalanced.

After learning the locations of the correct corners in the initial training trial, participants proceeded to training phase. In the first training phase they received trials in the same room as initial training and the trials were organized into blocks of five trials each. On each trial, they were asked to choose one of the two correct corners. Each trial was followed with feedback, as described above. To proceed to the next training phase, participants had to choose one of the two correct corners on four of the five trials in one block. If participants did not meet this criterion, they received more blocks of five trials until they passed.

The second phase of training showed the same environment to participants, and the trials were also organized into blocks of five trials each. But on three of the five trials (randomly determined), participants received an uninformative feedback (an OK sign with a neutral click sound) regarding their choice, regardless of whether it was correct or incorrect. The criterion to pass the second phase was the same as the first; if participants were correct in four of five trials in one block, they proceeded to the testing phase.

In the testing phase, participants received five blocks of trials that included each of the four tests (Control, Height Only, Distance or Angles Only, and Conflict) in a randomized order within each block. All test trials were followed by uninformative feedback.

\section{Results and discussion}

Preliminary analyses The performance in each test was calculated separately for each participant. The responses in Conflict tests were coded in terms of the correct corners according to height cues. Figure 2 shows the average correct proportions for each test in each environment.

Because there were no absolute correct corners in Conflict tests (i.e., the responses in Conflict tests were correct in terms of either horizontal or height cues), the responses for this test were analyzed separately.

Preliminary analyses on the data from the remaining tests revealed that sex, location of correct corners, and order of sessions (tested rectangular environment first or rhombus environment first) had no effect on the proportion of correct responses (all $p \mathrm{~s}>.10$ ). As a result, subsequent analyses were conducted with data collapsed across these factors.

To examine how participants used the horizontal cues and height cues in the rectangular and rhombus environments, further analyses were conducted to explore the performance in each test of the two environments separately. Bonferroni's correction for multiple $t$ tests was used if applicable (corrected $\alpha=.05 /$ number of $t$ tests).

Distance vs. height: Training in the rectangular room The correct proportion in each test of the rectangular environment was compared with chance level (i.e., 0.5) by one-sample $t$ test (see Fig. 2). The accuracy in Distance Only, Height Only, and Control tests was significantly higher than chance level (all $p$ s $<.001<$ corrected $\alpha$, Cohen's $d \mathrm{~s}>1.76$ ), indicating successful use of distance cues or height cues alone or together.

To compare the accuracy in the three tests (Control, Distance Only, Height Only), a repeated-measures ANOVA with accuracy as the dependent variable was conducted and showed significant difference among the three tests, $F(1.41$, $53.75)=6.39, p=.008, \eta_{\mathrm{p}}{ }^{2}=.14$. Further analysis showed no difference between the accuracy in Distance Only test and Height Only test, $t(38)=-0.39, p=.697$, Cohen's $d=0.09$. Accuracy in the Control test was significantly higher than the Distance Only test and Height Only test (both $t \mathrm{~s}>3.51, p \mathrm{~s}<$ $.002<$ corrected $\alpha$, Cohen's $d \mathrm{~s}>0.79$ ).

Angles vs. height: Training in the rhombus room The correct proportion in each test of the rhombus environment was compared with chance level (i.e., 0.5) by one-sample $t$ tests separately (see Fig. 2). The accuracy in the Angles Only, Height Only, and Control tests were significantly higher than chance level (all $p \mathrm{~s}<.001<$ corrected $\alpha$, Cohen's $d \mathrm{~s}>1.05$ ), indicating successful use of angle cues or height cues alone or together.

A repeated-measures ANOVA with accuracy in three tests (Control, Angles Only, Height Only) as the dependent variable showed significant difference, $F(1.28,48.71)=8.27, p=$ $.003, \eta_{\mathrm{p}}{ }^{2}=.18$. Further analysis showed no difference between the accuracy in the Angles Only test and Height Only test, $t(38)=1.58, p=.121$, Cohen's $d=0.36$. Accuracy in the Control test was significantly higher than in the Angles Only test and Height Only test (both $t \mathrm{~s}>2.91, p \mathrm{~s}<.007<$ corrected $\alpha$, Cohen's $d \mathrm{~s}>0.65$ ).

Conflict tests In the rectangular environment, participants chose the corners that were correct according to height cues significantly more often than would be expected by chance, $t(38)=2.63, p=.012$, Cohen's $d=0.59$, indicating that height cues dominated their choice. However, in the rhombus environment, choice of the corners that were correct according to height cues was not significantly different from chance level, $t(38)=0.41, p=.682$, Cohen's $d=0.09$, indicating that the participants did not show a systematic preference for either angular cues or height cues.

A mixed ANOVA with order of sessions as the betweensubjects variable and testing environment as the withinsubjects variable revealed a significant main effect of testing environment: Participants chose correct corners according to 
height more often in the rectangular environment than in the rhombus environment, $F(1,37)=4.68, p=.037, \eta_{\mathrm{p}}{ }^{2}=.11$. There was also a significant interaction between testing environment and order of sessions, $F(1,37)=5.23, p=.028, \eta_{\mathrm{p}}{ }^{2}=$ .12. An analysis of simple effects showed that the participants who first received the rhombus environment chose according to height cues more often in the rectangular environment than in the rhombus environment, $t(19)=4.20, p<.001$, Cohen's $d=1.33$. However, the participants who first received the rectangular environment showed no significant difference in how often they chose corners that were correct according to height cues in the two environments, $t(18)=0.07, p=.944$, Cohen's $d=0.02$.

We speculated that the interaction between testing environment and order of sessions might be because the relative salience of height increased in the second environment compared with that in the first environment. Height cue was presented in both environments whereas the other cues (angle and distance) changed across the two environments. Therefore, the relative salience of height in the second environment increased compared with that in the first environment. Participants chose correct corners according to height more often in the rectangular environment than in the rhombus environment. This might have enhanced the effect that participants chose correct corners according to height more often in the rectangular environment than in the rhombus environment when the rectangular room was the second testing environment but might have counterbalanced the testing environment effect when the rhombus room was the second testing environment. To examine this speculation, a mixed ANOVA that included testing condition (first or second) and order of sessions (whether the first condition was the rectangular or the rhombus environment) as independent variables revealed a significant main effect of testing condition: Participants chose correct corners according to height cues more often in their second testing condition than in their first one, $F(1,37)=5.23$, $p=.028, \eta_{\mathrm{p}}{ }^{2}=.12$.

We also examined the consistency of strategy use by individual participants on the Conflict tests. If a participant chose according to the same strategy (i.e., either height cues or horizontal cues) on at least four out of five test trials, they were classified as using that strategy. In rectangular environment, $61.5 \%$ of the participants used a height strategy and $25.6 \%$ used a horizontal strategy. In the rhombus environment, $38.5 \%$ of the participants used a height strategy and $48.7 \%$ used a horizontal strategy. Only 10 participants (25.6\%) maintained a height strategy and five participants (12.82\%) maintained a horizontal strategy in both environments.

The results from the Conflict test in Experiment 1 suggest that horizontal cues were not preferred over height cues in either environment. Instead, in the rectangular environment participants more often relied on height cues than on horizontal distance cues.
Why might participants have used height cues more than horizontal cues in the rectangular environment, but not in the rhombus environment? It might be possible that in the rectangular environment, participants tended to choose height cues because they noticed the height difference of the walls before they paid attention to the distance cues. Participants were not asked to view all four corners before they made a choice in either environment, so they could make a response immediately once they started a new trial. The field of view (FOV) of the VR was relatively narrow compared to the FOV from real eyes. Through the goggle of VR, it was difficult to see two corners at the same time without physically turning their bodies. Therefore, height difference of walls could easily be detected by taking a look at only one corner, and this information could be used to make a choice immediately, whereas the distance cues could not be detected without turning around to compare the lengths of at least two walls. Therefore, using horizontal cues might require participants to exert relatively more effort. Using height cues may therefore be an easier strategy in our rectangular environment because participants could choose based on height cues at their first sight of a corner.

In the rhombus environment, however, the differences between angles $\left(60^{\circ}\right.$ vs. $\left.120^{\circ}\right)$ may have been sufficiently salient that participants could determine this horizontal property without turning around. This could account for why participants chose according to horizontal cues more often in the rhombus environment than in rectangular environment.

On the Conflict tests, we also found a significant interaction between the test environment and the order of sessions: Only participants who experienced the rhombus environment first showed more choice according to height cues in the rectangular than in the rhombus environment. It is possible that the participants who first received the rhombus environment used height cues more because the horizontal cues changed across the two environments, whereas height cues were constant. To exclude the potential influence of such an experience, we tested participants only in one environment in Experiment 2.

\section{Experiment 2}

Experiment 2 used the same rectangular environment as in Experiment 1, but the procedure was modified to require participants to look at all corners prior to making a choice. This change was designed to exclude the possible contribution of insufficient detection of the environment and hasty choice to the conflict test results in the rectangular environment. In addition, participants were tested only in the rectangular environment, thus precluding any contribution of prior exposure to the height cues. Testing in only one environment also allowed us to include more 
than five trials with each test. Therefore, in Experiment 2, five more trials for each test (i.e., five more blocks) were added. Because no feedback was provided in testing, retraining was provided after five testing blocks to refresh participants on the contingencies.

\section{Method}

Participants Forty-two students (20 males, 22 females, age range $=17-26$ years, $M=19.21$ years) from the University of Alberta participated in Experiment 2. Three additional participants were excluded because of low performance (lower than $80 \%$ correct) in the Control test. All of the participants received course credits for their participation.

Apparatus, design, and procedure Apparatus, design, and procedure were exactly the same as in Experiment 1, with four exceptions. First, participants only received trials in the rectangular environment within one session. Second, on each trial, participants had to point the wand at each of the corners without clicking so that they saw all four corners before making a choice. Third, on each trial, participants were asked to choose two corners, and both of the choices were recorded. Fourth, after the testing phase with five blocks identical to Experiment 1 , participants received two training phases again, which were exactly the same as in the first and second training phases and also had the same passing criterion. Afterwards, participants were tested in five more blocks, which were the same as the first testing phase. Therefore, in total, participants received 10 trials for each test.

\section{Results and discussion}

The accuracy in Distance Only, Height Only, and Control tests and the proportion of choices to distance correct corners in Conflict test was compared with chance level (i.e., 0.5 ) by a one-sample $t$ test, and the results are shown in Fig. 3. The accuracy in Distance Only, Height Only, and Control tests was significantly higher than chance level (all $p$ s $<.001<$ corrected $\alpha$, Cohen's $d \mathrm{~s}>3.02$ ), indicating successful use of distance cues or height cues alone or together.

A repeated-measures ANOVA with correct proportions in the three tests (Control, Distance Only, Height Only) as the dependent variable was conducted. Consistent with the results in Experiment 1, there was a significant effect of test, $F(1.35$, $55.25)=8.27, p=.003, \eta_{\mathrm{p}}{ }^{2}=.17$, and further analysis showed no difference between the accuracy in the Distance Only test and the Height Only test, $t(41)=-0.69, p=.697$, Cohen's $d=$ 0.15 . Accuracy in the Control test was significantly higher than in either the Distance Only test or the Height Only test (both $t \mathrm{~s}>4.20, p \mathrm{~s}<.001<$ corrected $\alpha$, Cohen's $d \mathrm{~s}>0.91$ ).

In the Conflict test, different from the results in the rectangular environment in Experiment 1, the proportion of choices
Experiment 2

Distance vs height
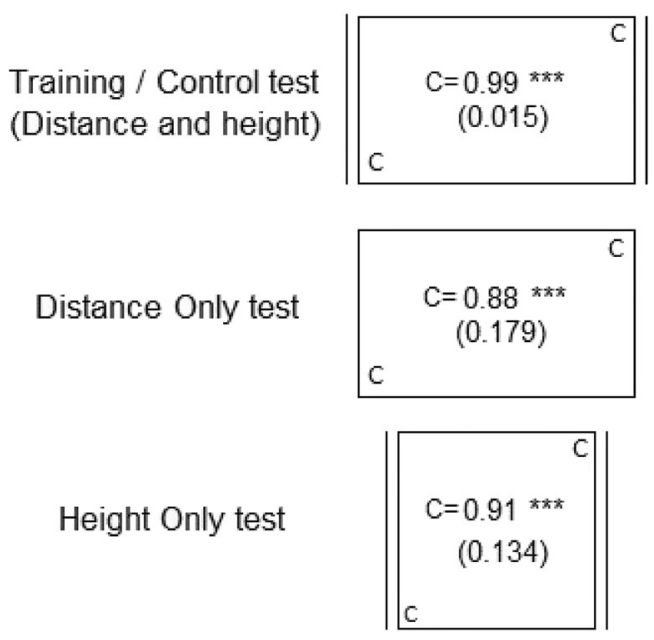

Conflict test

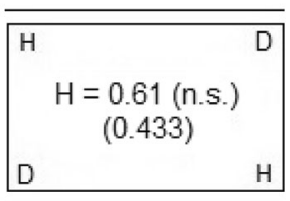

Fig. 3 The top view of the experimental environments and the average choice proportion in each test in Experiment 2 compared with chance level $(0.5)$ in each test (one-sample $t$ test: $* * * p<.001$ $<$ corrected $\alpha$; ${ }^{*} p<.05<$ corrected $\alpha$; n.s. $p>.05$ ). The double line indicates a high wall, and the single line indicates a low wall. The letter $C$ indicates a correct corner. In the conflict test, the letter $H$ indicates a correct corner according to height cues. For simplicity, the correct corners are illustrated for the case in which the corners on the left to a short wall were correct. The numbers in parentheses are standard deviation in that test

to the height correct corner was not significantly different from chance level, $t(41)=1.71, p=.094$, Cohen's $d=0.37$. Across the 10 Conflict test trials, $56.4 \%$ of the participants chose according to height cues, and $31.0 \%$ chose according to horizontal cues on at least $80 \%$ of the trials.

The results in Experiment 2 suggest that, although the potential influences on response were controlled, participants still did not show preference for the horizontal information (distance) in the Conflict test.

\section{General Discussion}

The two experiments indicated that after training, in which both horizontal and height cues provided equally reliable cues for reorientation, participants were able to use either cue alone to reorient; this was the case whether the horizontal cues were presented as either distance information (via a rectangular environment) or angle information (via a rhombus environment). Although performance with either cue alone was well 
above chance, it was slightly, but significantly, lower than performance on the Control tests with both cues. This reduction in accuracy could be due to the novelty of having only one cue present, or it could reflect the benefit of combining multiple cues in the Control test. Our results cannot distinguish these possibilities, but they clearly show that adult humans can readily use the vertical cue provided by wall height to reorient. This use of vertical cues is consistent with the findings that adults can use slope cues (Nardi et al., 2011) and that older children can use height cues provided by a slanted ceiling ( $\mathrm{Hu}$ et al., 2015).

Our study showed that when horizontal and height cues were presented in conflict, participants did not show a preference for the horizontal cues. In particular, participants who had previously experienced the rhombus environment showed a clear preference for using height cues over the distance cues when they were subsequently trained and tested in the rectangular environment. In Experiment 2, participants who received more extensive testing in just the rectangular environment still did not make more choices according to distance cues in the Conflict test. The lack of dominance by horizontal cues seems inconsistent with the findings in human children (Hu et al., 2015). Although this inconsistency may reflect developmental differences, there are also several procedural differences between our study and $\mathrm{Hu}$ et al. (2015) that could have led to the different results. Notably, in our study, the vertical cues provided symmetric directional information (i.e., two high walls) whereas the apparatus in $\mathrm{Hu}$ et al. (2015) provided only one high wall; this may have made the height cues more salient in our study. Our virtual environment also provided no ceiling, which eliminated any angular information from the slanted ceiling and thus may have provided more purely vertical information. In our study, the participants stood in the center of the virtual room to choose corners and did not move within the virtual environment, whereas the children in $\mathrm{Hu}$ et al. (2015) walked to the corner of enclosure to make a choice. It is possible, therefore, that children received more information about the horizontal cues of the enclosure through their walking, and this may have led to the better performance with horizontal cues than with height cues. However, a previous study found that although restriction of movement made it less likely that young children could use a feature, it did not affect their use of horizontal geometry (Learmonth et al., 2008). In addition, differences in room size may have played a role. It is possible that the walls in $\mathrm{Hu}$ et al. (2015) were too close to the children and all too high for the children to notice and use. The largest room in Hu et al. (2015) was $1.8 \mathrm{~m} \times 1.8 \mathrm{~m}$, whereas in our study the smallest virtual rooms were more than double that size, which may have made the height cues easier to perceive.

Another major difference is that our study used VR environments whereas Hu et al. (2015) used real rooms. Viewing VR environments usually is functionally the same as viewing real rooms, especially with an immersive, interactive VR like that used in the current study except that distances and sizes are usually underperceived in virtual environments (Kelly, Hammel, Sjolund, \& Siegel, 2015). Moreover, our results on both the Distance Only and Height Only tests suggest that participants successfully encoded horizontal and vertical cues with the VR environment and could use single types of cues to reorient. It is possible, however, that the FOV in our study influenced the salience of the cues in the encoding process. Previous studies on FOV suggest that narrower FOV can interfere with the encoding of horizontal geometry in reorientation (Sturz, 2014; Sturz, Kilday, \& Bodily, 2013). For example, one study compared Horizontal $\times$ Vertical FOV settings of $50^{\circ} \times$ $32^{\circ}$ with the settings of $100^{\circ} \times 74^{\circ}$. The FOV in our study $\left(44^{\circ}\right.$ $\times 35^{\circ}$ ) was even narrower on the horizontal dimension. As we discussed in Experiment 1, the FOV in our study might have increased the effort required to view the horizontal geometry in the rectangular environment. In contrast, on the vertical dimension, participants could see the difference between the heights of the walls more easily than the lengths of the walls. Therefore, the potential influence of narrow FOV might only lie on the encoding of horizontal geometry. It may also be one reason for why participants did not follow horizontal cues in the Conflict test. Although our study was not designed to provide specific tests to distinguish between the geometric module theory and the adaptive combination theory of spatial reorientation, our findings nevertheless have implications for these theories. The geometric module theory (Spelke et al., 2010) defines Euclidean geometry as the shapes of the extended surfaces that form the borders of the traversable layout, which restricts the distance information to the Euclidean plane. However, this theory seems to be silent with respect to the vertical dimension of space. Our study suggests that, at least under some circumstances, the vertical dimension of space may be as important for reorientation as is horizontal geometry.

The adaptive combination theory (Newcombe \& Huttenlocher, 2006; Twyman et al., 2013) suggests that use of cues for reorientation is more a function of the salience, reliability, and familiarity of a cue than on fixed absolute properties of the cue, such as whether it is a geometric or a featural cue. Our results fit well with this theory. First, as we have discussed, difference in relative salience on perceiving the cues may account for why horizontal geometry did not dominate over height cues in our study, as was found in $\mathrm{Hu}$ et al. (2015). Second, the familiarity and stability of the cues may have played a role in our study. In Experiment 1, the vertical height cues appeared to dominate over horizontal geometric cues (distance) in the rectangular environment, but the interaction with order suggested that this dominance occurred primarily when participants were first exposed to the rhombus environment. This dominance of height cues for this particular group of participants may have reflected the fact that the same height cues were present in both environments; therefore, after 
training in the rhombus environment, height cues would be stable and familiar in the rectangular environment whereas the horizontal cues changed between environments. An effect of prior experience is consistent with previous findings on the use of features and horizontal geometry (Learmonth et al., 2008; Ratliff \& Newcombe, 2008; Twyman, Friedman, \& Spetch, 2007). In Experiment 2, when participants were tested in only the one environment so that the height and horizontal cues were equally stable and familiar, the vertical height cues did not show obvious dominance over horizontal geometry. Thus, consistent with the adaptive combination theory, the relative dominance of the cues was labile.

Although height, like horizontal distance, contains metric information, we cannot conclude that vertical distance functioned as a geometric cue. It is possible that our height cue functioned instead like a featural cue. Indeed, just as the angular information might be visually salient from one view without looking around, the height change at the corner would be visible from a single view. Sturz et al. (2012) argued that angles function more like featural cues, and the same may be the case for our height cues. Interestingly, the distinction between featural cues and geometric cues, and the reasons for dominance of geometric information, have often been discussed in terms of the usefulness of each type of cue in nature. That is, although the geometric layout of environments is typically stable over an organism's lifetime, surface features, such as color, can change over seasons or even lighting conditions. Thus, regardless of whether height cues function as geometric or featural cues, they would be expected to be used for orientation based on functional considerations.

In conclusion, our results show that human adults can use wall height alone as a vertical cue to reorient in a virtual environment. When the height cues conflicted with horizontal properties of the environment, human adults did not prefer horizontal information (distance or angle) over height cues, and in some cases gave priority to the height cues. The current results seem consistent with the adaptive combination theory. Our results supplement and extend the existing findings about the use of geometry in reorientation and suggest that, at least under some circumstances, nontraversable (vertical) distance can be a salient cue for reorientation, at least by adults.

Author note This research was funded by the Natural Sciences and Engineering Research Council of Canada to Marcia L. Spetch and Weimin Mou. We thank Benson $\mathrm{Ng}$ for his contribution in data collection. We have no conflict of interest to declare.

\section{References}

Cheng, K. (1986). A purely geometric module in the rat's spatial representation. Cognition, 23, 149-178. doi:10.1016/0010-0277(86)90041-7

Cheng, K., Huttenlocher, J., \& Newcombe, N. S. (2013). 25 years of research on the use of geometry in spatial reorientation: A current theoretical perspective. Psychonomic Bulletin \& Review, 20, 10331054. doi:10.3758/s13423-013-0416-1

Cheng, K., \& Newcombe, N. S. (2005). Is there a geometric module for spatial orientation? Squaring theory and evidence. Psychonomic Bulletin \& Review, 12, 1-23. doi:10.3758/BF03196346

Graham, P., \& Cheng, K. (2009). Which portion of the natural panorama is used for view-based navigation in the Australian desert ant? Journal of Comparative Physiology A: Neuroethology, Sensory, Neural, and Behavioral Physiology, 195, 681-689. doi:10.1007/s00359-009-0443-6

Hermer, L., \& Spelke, E. S. (1994). A geometric process for spatial reorientation in young children. Nature, 370, 57-59.

Hermer, L., \& Spelke, E. (1996). Modularity and development: The case of spatial reorientation. Cognition, 61, 195-232.

Holbrook, R. I., \& Burt de Perera, T. (2009). Separate encoding of vertical and horizontal components of space during orientation in fish. Animal Behaviour, 78, 241-245. doi:10.1016/j.anbehav.2009.03.021

Holbrook, R. I., \& Burt de Perera, T. (2011). Three-dimensional spatial cognition: Information in the vertical dimension overrides information from the horizontal. Animal Cognition, 14, 613-619. doi:10.1007/s10071-011-0393-6

Holmes, C. A., Nardi, D., Newcombe, N. S., \& Weisberg, S. M. (2015). Children's use of slope to guide navigation: Sex differences relate to spontaneous slope perception. Spatial Cognition \& Computation, 15, 170-185. doi:10.1080/13875868.2015.1015131

Hu, Q., Zhang, J., Wu, D., \& Shao, Y. (2015). Is height a core geometric cue for navigation? Young children's use of height in reorientation. Journal of Experimental Child Psychology, 130, 123-131. doi:10.1016/j.jecp.2014.10.003

Hupbach, A., \& Nadel, L. (2005). Reorientation in a rhombic environment: No evidence for an encapsulated geometric module. Cognitive Development, 20, 279-302. doi:10.1016/j.cogdev.2005.04.003

Kelly, J. W., Hammel, W., Sjolund, L. A., \& Siegel, Z. D. (2015). Frontal extents in virtual environments are not immune to underperception. Attention, Perception, \& Psychophysics, 77, 1848-1853. doi:10.3758/s13414-015-0948-8

Learmonth, A. E., Nadel, L., \& Newcombe, N. S. (2002). Children's use of landmarks: Implications for modularity theory. Psychological Science, 13, 337-341. doi:10.1111/j.0956-7976.2002.00461.x

Learmonth, A. E., Newcombe, N. S., \& Huttenlocher, J. (2001). Toddlers' use of metric information and landmarks to reorient. Journal of Experimental Child Psychology, 80, 225-244. doi:10.1006/jecp.2001.2635

Learmonth, A. E., Newcombe, N. S., Sheridan, N., \& Jones, M. (2008). Why size counts: Children's spatial reorientation in large and small enclosures. Developmental Science, 11, 414-426. doi:10.1111 j.1467-7687.2008.00686.x

Lee, S. A., \& Spelke, E. S. (2008). Children's use of geometry for reorientation. Developmental Science, 11, 743-749. doi:10.1111/j.14677687.2008.00724.x

Lee, S. A., \& Spelke, E. S. (2011). Young children reorient by computing layout geometry, not by matching images of the environment. Psychonomic Bulletin \& Review, 18, 192-198. doi:10.3758 /s13423-010-0035-z

Lee, S. A., Spelke, E. S., \& Vallortigara, G. (2012). Chicks, like children, spontaneously reorient by three-dimensional environmental geometry, not by image matching. Biology Letters, 8, 492-494. doi:10.1098/rsbl.2012.0067

Legge, E. L. G., Wystrach, A., Spetch, M. L., \& Cheng, K. (2014). Combining sky and earth: Desert ants (Melophorus bagoti) show weighted integration of celestial and terrestrial cues. Journal of Experimental Biology, 217(23), 4159-4166. doi:10.1242/jeb.107862

Lubyk, D. M., Dupuis, B., Gutiérrez, L., \& Spetch, M. L. (2012). Geometric orientation by humans: Angles weigh in. Psychonomic Bulletin \& Review, 19, 436-442. doi:10.3758/s13423-012-0232-Z

Lubyk, D. M., Spetch, M. L., Zhou, R., Pisklak, J., \& Mou, W. (2013). Reorientation in diamond-shaped environments: Encoding of 
features and angles in enclosures versus arrays by adult humans and pigeons (Columbia livia). Animal Cognition, 16, 565-581. doi:10.1007/s10071-012-0594-7

Nardi, D., \& Bingman, V. P. (2009). Pigeon (Columba livia) encoding of a goal location: The relative importance of shape geometry and slope information. Journal of Comparative Psychology, 123, 204216. doi:10.1037/a0015093

Nardi, D., Newcombe, N. S., \& Shipley, T. F. (2011). The world is not flat: Can people reorient using slope? Journal of Experimental Psychology: Learning, Memory, and Cognition, 37, 354-367. doi:10.1037/a0021614

Nardi, D., Nitsch, K. P., \& Bingman, V. P. (2010). Slope-driven goal location behavior in pigeons. Journal of Experimental Psychology: Animal Behavior Processes, 36, 430-442. doi:10.1037/a0019234

Nardini, M., Atkinson, J., \& Burgess, N. (2008). Children reorient using the left/right sense of coloured landmarks at 18-24 months. Cognition, 106, 519-527. doi:10.1016/j.cognition.2007.02.007

Newcombe, N. S., \& Huttenlocher, J. (2006). Development of spatial cognition. In D. Kuhn \& R. Siegler (Eds.), Handbook of child psychology: Vol. 2. Cognition, perception, and language (6th ed., pp. 734-776). New York, NY: John Wiley.

Ratliff, K. R., \& Newcombe, N. S. (2008). Reorienting when cues conflict: Evidence for an adaptive-combination view. Psychological Science, 19, 1301-1307. doi:10.1111/j.1467-9280.2008.02239.x

Spelke, E., Lee, S. A., \& Izard, V. (2010). Beyond core knowledge: Natural geometry. Cognitive Science, 34, 863-884. doi:10.1111 j.1551-6709.2010.01110.x
Sturz, B. R. (2014). Modeling a role of field of view in the extraction of geometric cues during reorientation. Frontiers in Psychology, 5, 1-3. doi:10.3389/fpsyg.2014.00535

Sturz, B. R., Forloines, M. R., \& Bodily, K. D. (2012). Enclosure size and the use of local and global geometric cues for reorientation. Psychonomic Bulletin \& Review, 19, 270-276. doi:10.3758/s13423011-0195-5

Sturz, B. R., Kilday, Z. A., \& Bodily, K. D. (2013). Does constraining field of view prevent extraction of geometric cues for humans during virtual-environment reorientation? Journal of Experimental Psychology: Animal Behavior Processes, 39, 390-396. doi: $10.1037 / \mathrm{a} 0032543$

Twyman, A. D., Friedman, A., \& Spetch, M. L. (2007). Penetrating the geometric module: Catalyzing children's use of landmarks. Developmental Psychology, 43, 1523-1530. doi:10.1037/00121649.43.6.1523

Twyman, A. D., Nardi, D., \& Newcombe, N. S. (2013). Two fields are better than one: Developmental and cognitive perspectives on underatnding spatial reorientation. Comparative Cognition \& Behavior Reviews, 8, 78-97. doi:10.3819/ccbr.2013.80005

Twyman, A. D., \& Newcombe, N. S. (2010). Five reasons to doubt the existence of a geometric module. Cognitive Science, 34, 1315-1356. doi:10.1111/j.1551-6709.2009.01081.x

Wystrach, A., Beugnon, G., \& Cheng, K. (2011). Landmarks or panoramas: What do navigating ants attend to for guidance? Frontiers in Zoology, 8(21). doi:10.1186/1742-9994-8-21 